\title{
HIGH-RESOLUTION IN SITU HOLOGRAPHIC RECORDING AND ANALYSIS OF MARINE ORGANISMS AND PARTICLES (HOLOMAR) $^{*}$
}

\author{
J Watson ${ }^{\mathrm{a}}$ \\ V Chalvidan ${ }^{\mathrm{b}}$, JP Chambard ${ }^{\mathrm{b}}, \mathrm{G} \mathrm{Craig}^{\mathrm{a}}$, A Diard ${ }^{\mathrm{c}}$, GL Foresti ${ }^{\mathrm{d}}$, B Forre ${ }^{\mathrm{e}}$, S Gentili ${ }^{\mathrm{d}}$, PR Hobson ${ }^{\mathrm{f}}$,

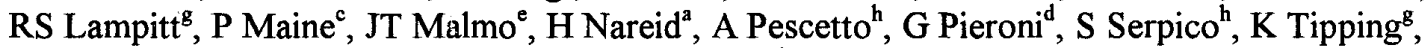 \\ A Trucco ${ }^{\text {h }}$
}

\author{
a Department of Engineering, Aberäeen University, Aberdeen AB24 3UE, Scotland \\ ${ }^{\mathrm{b}}$ Holo 3, 7 rue de General, Saint-Louis 68300, France \\ ${ }^{\circ} Q$ uantel, 17 Ave de l'Atlantique, 91941 Les Ulis, France \\ ${ }^{d}$ Dept of Mathematical \& Information (DIMI), University of Udine, via del Scienze 206, Udine 33100, Italy \\ ${ }^{\mathrm{e}}$ Nemko, Sem Saelands v.5, N-7034 Trondheim, Norway \\ Institute of Physical and Environmental Sciences, Brunel University, Uxbridge, Middlesex UB8 3PH, England

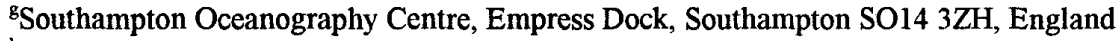 \\ ${ }^{\mathrm{h}}$ Dept of Biophysical \& Electronic Engineering (DIBE), University of Genova, via all'Opera Pia 11A, Genova I-16145, \\ Italy
}

\begin{abstract}
-
We report on the development of a fully-functioning, prototype, underwater holographic camera (holo-camera) for holographic recording of large-volumes of sea water containing marine plankton and seston within the upper water column. The overriding benefit of holographic imaging over other measurement techniques is that it allows non-intrusive and non-destructive, in-situ, recording of living organisms and inanimate particles in their natural environment. Because of the inherently high resolution of holography, its threedimensional imaging properties and the ability to perform "optical sectioning" on the image, it allows identification of particular organisms together with the extraction of size and relative positional information. This information, in turn, affords the ability to gain knowledge of the behaviour of marine biological communities, their relationship with each other and with the particles with which they interact.
\end{abstract}

\section{INTRODUCTION}

It is well known that a detailed knowledge of the distribution and dynamics of marine organisms and particles in the marine water column is crucial to our understanding of chemical cycles in aquatic systems [1-3]. The surface waters of oceans are rich in plankton which play a crucial role in sustaining biological productivity of the marine environment and, in turn, affect global carbon cycling. Oceans are also full of aggregates of other living and dead particles that also influence the cycling of materials and act as a focus for biological activity and help to control the diversity of the biosphere [1]. Marine aggregates, often referred to as marine snow, may vary in size from tens of microns to several millimetres and vary in structure depending on their mode of formation $[2,3]$.
Their frailty, wide size range and complex structure have complicated previous observations of aggregate particles.

Because of its particular properties, holographic recording and imaging of marine systems offers a unique tool to biologists for the analysis of such systems [4-8]. The overriding benefit of holographic imaging is that it allows non-intrusive and non-destructive recording, in-situ, of living, motile, organisms and inanimate particles in their natural environment. The inherently high resolution of holography, its three-dimensional imaging properties and the ability to perform "optical sectioning" on the replayed image, allows enumeration and identification of specific organisms together with their size, and three-dimensional spatial distribution. A simultaneous combination of a large depth-of-field and high-resolution of a hologram enables the recording of large volumes of small particles in a single exposure; compared to that of a photograph vastly more data can be recorded in a hologram. In addition, the wide dynamic range enables holographic images to yield information where photographs would possess exceedingly high levels of background noise. Low-noise is particularly valuable in recording semi-transparent particles of micron dimensions at low concentrations.

In our collaboration, we are developing a fully functioning, prototype, underwater holographic camera (holo-camera) to record large-volumes (up to $10^{5} \mathrm{~cm}^{3}$ ) of marine organisms (marine plankton and seston) within the upper water column. The HOLOMAR system will consist of two related

\footnotetext{
- Work funded by The European Commission under the MAST-III initiative (MAS3-CT97-0079).
} 
and inter-dependent facilities: a fully-configured underwater holo-camera (HOLOCAM) for recording the organisms, and a dedicated hologram replay and data analysis facility (HOLOSCAN). Specially developed image processing algorithms will allow identification of species at the family or genus level together with the ability to automatically extract information regarding shape, dimensions, relative position and measurement of local concentration and distribution of a variety of marine organisms. The entire system will be demonstrated and evaluated in a series of laboratory, tank, dockside and, if possible, sea trials.

The project involves eight Institutions from four partner countries of the EC and its affiliates. The partners (Table 1) were chosen because of their compatibility and coverage of the wide range of skills required of HoloMar.

Table 1: Partners in HoloMar

\begin{tabular}{|l|l|}
\hline PARTNER & ROLE IN HOLOMAR \\
\hline $\begin{array}{l}\text { Aberdeen Univ, Scotland } \\
\text { (Co-ordinator) }\end{array}$ & Holographic camera/optics design \\
\hline Brunel Univ. England & Hologram replay \\
\hline Holo 3, France & Emulsion evaluation \& calibration \\
\hline Quantel, France & Laser design \& manufacture \\
\hline Genova Univ, Italy & Data acquisition \& image enhancement \\
\hline Udine Univ, Italy & Image identification \& classification \\
\hline Nemko, Norway & Camera housing, control, plate transport \\
\hline $\begin{array}{l}\text { Southampton Oceanography } \\
\text { Centre, England }\end{array}$ & Submersible frame, hologram analysis \\
\hline
\end{tabular}

In this paper, we outline the concepts of holography as applied to the recording and analysis of marine organisms, we describe the development of the experimental facilities within HOLOMAR. Some holographic images of phyto- and zoo-plankton recorded in laboratory simulations are presented along with associated data extracted from the holograms.

\section{ADVANTAGES OF HOLOGRAPHY}

The conventional methods of gathering in situ data on plankton, and other suspended particles, are not well suited to observing their precise spatial relationship within a large volume on an individual scale. For example, electronic counting techniques tend to destroy the plankton and flocs and consequently any intact visual record. Another example is high-resolution photography, which resolves only a narrow depth of field per exposure. Hence, sampling a large volume takes a considerable time, over which the particle distribution may change.

Holography is unique in the respect that it non-destructively and non-intrusively records a volume of live plankton species and other organisms in their natural environment, while preserving the accurate spatial distribution and image fidelity of individual particles. Moreover, a pulsed hologram can capture a large volume of the water column in a single exposure of a few nanoseconds duration. Sequential holograms can record changes within the volume over a defined period of time. True threedimensional imaging of organisms allows retention of parallax and perspective information and a high image resolution is obtainable over a large depth-of-field. The wide recording dynamic range afforded by a hologram allows images to be captured that would otherwise be buried in noise. On replay of the holograms, particles can be systematically examined by interrogating the real image with a CCD camera or microscope mounted on computer controlled micropositioners. The ability to isolate individual planes of the image, and move the viewing plane through the volume to bring individual species into focus sets holography apart from more conventional forms of imaging.

Holography can aid the study of marine biological communities by facilitating the measurement of size and relative position of organisms, and species identification and classification at genus level. From this data the distribution of organisms and their interrelationships could be extracted.

\section{CONCEPTS OF HOLOGRAPHY}

Of the many possible forms of holography only two recording schemes, "in-line" (Fraunhofer) and "off-axis" (Fresnel), are generally useful for high-resolution in situ imaging and mensuration of aquatic particles.

\section{A. IN-LINE HOLOGRAPHY}

With in-line holography, the illuminating beam of coherent light propagates through the sample volume towards the holographic plate which records the interference between light diffracted by the object and the undiffracted portion of the illuminating beam [e.g. 8]. No spatially separate reference beam is used. The replayed hologram simultaneously forms two images located on the optic axis, which for a collimated beam are at equal distances on either side of the holographic plate.

The range of object size recordable on an in-line hologram approaches an upper limit that is set by the requirement to record in the far-field of the object, i.e. that satisfies the conditions of Fraunhofer recording:

$$
z>d^{2} / \lambda
$$


where $z$ is the object-to-film distance, $d$ is the maximum dimension of the object to be recorded and $\lambda$ is the wavelength of the recording beam. The particular benefits of in-line holography are its geometrical simplicity, the minimisation of laser coherence and energy requirements, and the very high resolution capabilities over a large depthof-field. Experimentally it has been demonstrated (see for example [8]) that good holograms can be recorded on film as well as on glass plates.

Because in-line holograms need a significant amount of undiffracted reference light for successful recording high concentrations of particles give rise to strong speckle noise [9]. This sets an upper limit, for the HOLOCAM design presented here, of a few hundred particles of around $20 \mu \mathrm{m}$ dimension per cubic centimetre, on the concentrations that can be studied with the in-line technique.

\section{B. OFF-AXIS HOLOGRAPHY}

By contrast with in-line, off-axis holography records the interference between diffuse light reflected from the scene and a spatially separate reference beam that meets the plate at a finite angle. Consequently, off-axis holography is usually applied to primarily opaque subjects of large volume whereas in-line holography finds application for essentially transparent object scenes. Although the geometry is more complex off-axis holography is more flexible in the illumination and reference beam parameters that can be used to record particles in a volume of water [e.g. 4]. Diffuse front illumination allows larger population concentrations of particles to be recorded, providing that the particles reflect sufficient light to the film in order to form a holographic image. Since subjects can be illuminated at any suitable angle, and even from multiple angles and directions, subject visibility within the volume can be optimised. Off-axis holography may even provide a more complete record than the in-line case, by providing information on the structure of the object being recorded. Because Fresnel diffraction, rather than Fraunhofer, dominate the recording process, larger particle sizes than that of the in-line geometry can be recorded. However, there is a lower limit to the resolution that is set primarily by speckle and the physical constraints of the optical set-up.

Previous experimental results have demonstrated that resolutions of approximately $40 \mu \mathrm{m}$ (e.g. for a thin crack [4]) are possible for holograms of metal components located in clear water. Image formation for these larger particles is subject to the coherence length limitations of the laser and the availability of sufficient reflected light from the particle at the hologram plane.

\section{HOLOGRAMS OF OBJECTS IN WATER}

Because our interest is in the recording of objects in water, the water itself may be expected to influence the quality of the images produced. An increase in the overall turbidity of the water will adversely affect both techniques and would be expected to create a noise background that will reduce image fidelity. Holograms of particles recorded directly in water will also undergo a refractive index change when replayed in air. This will increase the influence of optical aberrations present in the reconstructed image. For in-line hologrammetry, only a small amount of spherical aberration is introduced since both reference and object beam angles are normal to the recording plane [10]. In the off-axis case, the resulting refractive index mismatch does produce significant aberrations, most noticeably astigmatism and coma, which increases with the field angle of the subject in the reconstructed image. Previous work $[11,12]$ on the origins of image aberrations resulting from refractive index mismatch suggests that image degradation can be minimised by replaying in air at the effective wavelength of the beam in water (i.e. a replay wavelength equal to the construction wavelength divided by the refractive index).

Typically, if recorded in water at a wavelength of $694 \mathrm{~nm}$ (ruby laser), the holograms should be replayed at $522 \mathrm{~nm}$ in air (in practice replaying at the $514 \mathrm{~nm}$ argon line is a good compromise). In this work, we will record using a frequency-doubled Nd-YAG laser $(532 \mathrm{~nm})$, The ideal compensation replay wavelength is then $400 \mathrm{~nm}$. However, no suitable laser lines exist in this region. We have opted to replay preferentially at the $\mathrm{HeCd}$ wavelength of $442 \mathrm{~nm}$ although the blue argon line of $457 \mathrm{~nm}$ would also be acceptable. However, complete correction assumes that the entire holographic system be located in water. Since this is both impractical and undesirable, the holograms are recorded with the holographic plate in air. Aberration balancing can still be accomplished if the hologram to window separation in the recording geometry is about a fifth of the window thickness $[11,12]$. For a window thickness of $30 \mathrm{~mm}$, an air gap of around $6 \mathrm{~mm}$ is optimum. However moving to a larger air gap of $120 \mathrm{~mm}$ also shifts the optimum replay wavelength to longer values.

\section{DESCRIPTION OF FACILITIES}

The holo-camera will consist of four elements: a laser, holographic optics, water-tight pressurised container and a support frame. Added to this will be the necessary control and power facilities. The self-contained reconstruction facility will comprise a laser, reconstruction optics and image processing and data-analysis instrumentation. 


\section{A. "HOLOCAM" PARAMETERS}

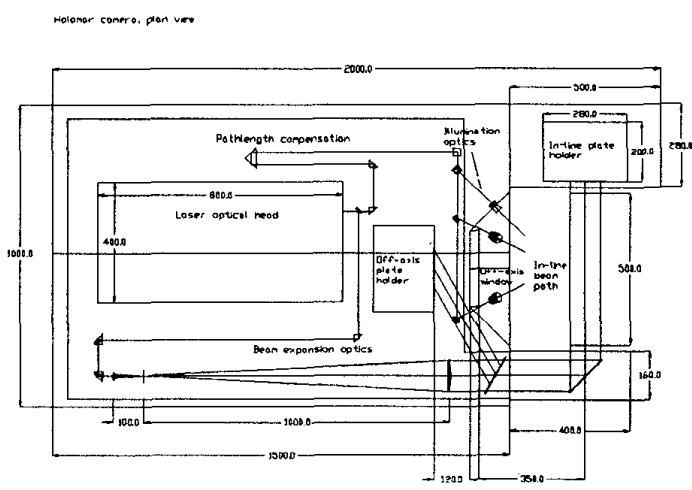

Figure 1: Schematic of proposed subsea HoloCamera

Holograms will be recorded of aquatic systems of up to $10^{5} \mathrm{~cm}^{3}$ volume, in-situ, using a pulsed laser. The chosen laser is a Q-switched, frequency-doubled Nd-YAG laser with a pulse duration of $8 \mathrm{~ns}$, an output energy of up to 650 $\mathrm{mJ}$ and a wavelength of $532 \mathrm{~nm}$ (in the green region of the light spectrum). Uniquely, the camera will incorporate the two basic holographic geometries, viz. in-line (Fraunhofer object in transmission) and off-axis (Fresnel - object in reflection). By using both geometries to simultaneously record overlapping volumes of water the camera will exploit the distinct advantages of each method. The in-line method can produce images of organisms in the range 5 to $250 \mu \mathrm{m}$, at concentrations up to a few thousand $\mathrm{cm}^{-3}$ at the smallest sizes. The off-axis method is better for larger organisms from about $100 \mu \mathrm{m}$ upwards at much higher concentration levels. Ultimately, the holo-camera will be capable of either ship deployment or attachment to a fixed buoy, will be remotely automated and controllable and designed to operate down to about $100 \mathrm{~m}$ depth. It will have the capacity to be equipped with other environmental sensors (such as temperature, salinity and pressure) if required. The overall dimensions of the camera will be around $1.2 \mathrm{~m}$ long by $500 \mathrm{~mm}$ diameter.

\section{B. "HOLOSCAN" PARAMETERS}

Although the recording of the holograms take place in water, replay of the image is carried out in the laboratory in air, using the real-image mode of reconstruction. The images are located in the space between the observer and the holographic film and are life size, fully threedimensional and retain all the parallax and perspective information of the original. A dedicated and automated reconstruction facility allows optimisation of the holographic image and computer-controlled precisiontranslation of a TV camera or measuring microscope through the image.

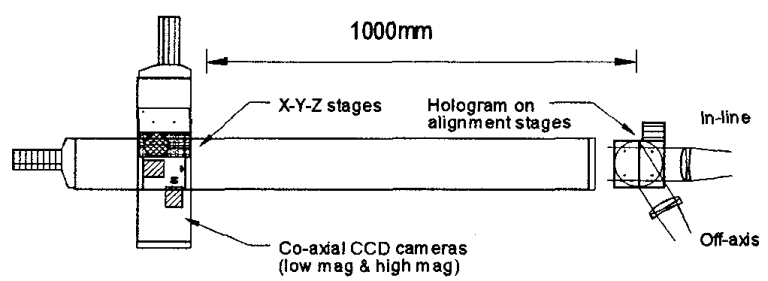

Figure 2: Schematic of proposed holographic replay facility

Specially developed image processing algorithms will allow identification of species at the family or genus level together with the ability to automatically extract information regarding shape, dimensions, relative position and measurement of local concentration and distribution of a variety of marine organisms. Figure 3 shows a digitally processed image of a phytoplankton organism (ceratium). The image was first extracted from an in-line hologram, then histogram manipulation, noise cleaning and binarisation by adaptive thresholding was performed by digital image processing techniques as a pre-cursor to classification by neural network methods.

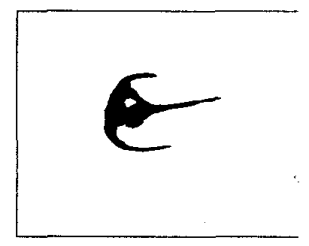

Figure 3: Digitally processed holographic image

\section{EXPERIMENTAL VALIDATION}

Experimental validation has been carried out in the laboratory on a series of calibrated targets and living marine organisms. Using in-line holography, cultured samples of freshwater biotic particles (Asterionella formosa and Tetrahymena pyriformis) were clearly resolved to better than $10 \mu \mathrm{m}$. Figure 4 shows images of asterionella recorded in a single in-line hologram. The middle picture is a detail from the left-hand image and shows a single organism about $70 \mu \mathrm{m}$ long. Also shown in Fig 4 is an image of the dinoflagellate Ceratium (right hand photo) recorded in a field sample of marine plankton. This organism is about $200 \mu \mathrm{m}$ long. 


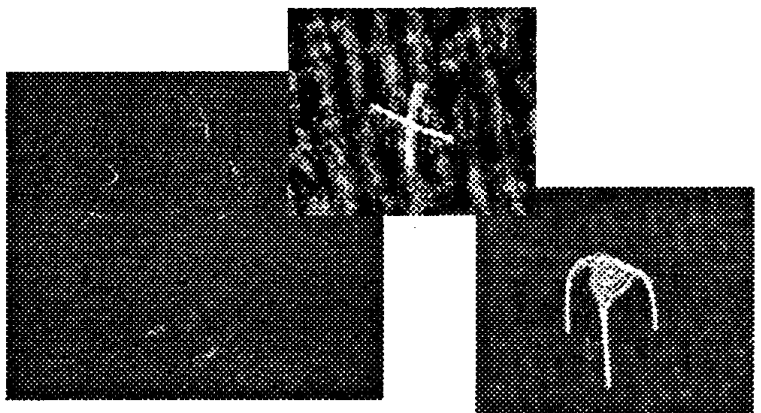

Figure 4: Images from an in-line hologram of asterionella

Figure 5 shows the three-dimensional co-ordinates of 601 individual diatoms (Asterionella), in a sub-volume of 98 $\mathrm{cm}^{3}$ of the total imaged volume of $2400 \mathrm{~cm}^{3}$ as measured from a single in-line hologram.

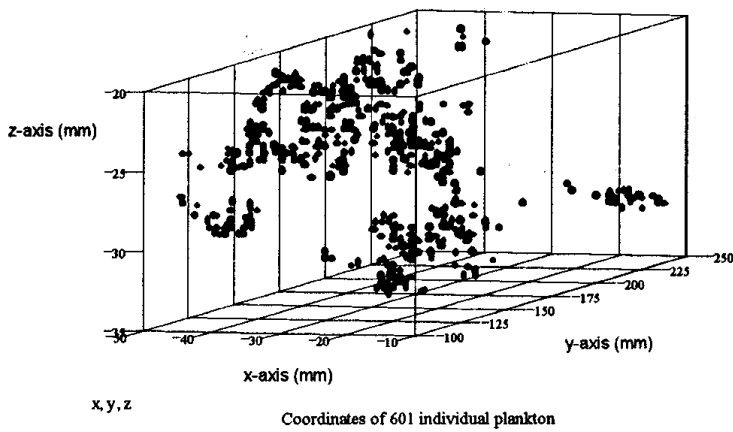

Figure 5: Position map of individual organisms in a single hologram

The reproducibility is better than $10 \mu \mathrm{m}$ in each axis. These co-ordinates were measured using an earlier version of our replay facility. The depth co-ordinates have been scaled to take into account the refractive index of the water, and the change of wavelength between recording and replay. Some of the particles had almost identical planar co-ordinates (in the transverse plane). However, they could still be clearly identified because of their separation in depth. For example two organisms, which had a lateral separation of only $84 \mu \mathrm{m}$, were easily identified as lying in two different planes, separated in depth by $4.28 \mathrm{~mm}$.

Off-axis holography provides lower resolution than in-line (around $100 \mu \mathrm{m}$ ), but records images of the larger macrozooplankton (e.g. Calenoid copepods) with dimensions of around $0.5 \mathrm{~mm}$ to $2 \mathrm{~mm}$. The off-axis method allows recording of particles in higher population concentrations than in-line (up to $200 \mathrm{~cm}^{-3}$ in water volumes of $2400 \mathrm{~cm}^{3}$ ).
Figure 6 shows two copepods recorded in the same hologram. The left organism is about $35 \mathrm{~mm}$ in front of, 3 $\mathrm{mm}$ higher and $2 \mathrm{~mm}$ to the left of the other. The out-offocus images of each organism can be seen in each picture. The copepods were present in concentrations of around $15 \mathrm{~cm}^{-3}$.

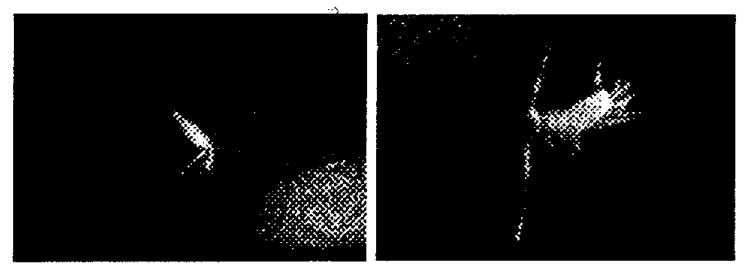

Figure 6: Copepods recorded in a single off-axis hologram

\section{ACKNOWLEDGEMENTS}

The authors wish to thank The European Commission for support of this work under the MAST-III initiative. Three of the authors (JW, PRH, RSL) wish also to thank the UK NERC for previous support of this work and all wish to thank their respective Institutions for giving them the encouragement and facilities to make this work possible.

\section{REFERENCES}

1. Amy., P.S., Caldwell, B.A., Soelder, A.H., Morita, R.Y. \& Albright, L.J. (1987) "Microbial activity and ultrastructure of mineral based marine snow from Howe Sound, British Columbia." Can. J. Fish. Aquat. Sci. 44: 1135-1142

2. Lampitt, R., Hillier, W.R. \& Challenor, P.G. (1993) "Seasonal and diel variation in the open ocean concentration of marine snow aggregates." Nature, 362: 737-739

3. Rogerson, A. \& Laybourn-Parry, J. (1992) "Aggregate dwelling protozooplankton communities in estuaries." Arch. Hydrobiol. 4: 411 422

4. Watson, J. (1989) "Hologrammetry and its applications." J. Imaging Tech., 15: $38-46$

5. Knox, C. (1966) "Holographic microscopy as a technique for recording dynamic microscopic subjects" Science, 153: $989-90$

6. Carder, K.L. et al., (1982) "In situ holographic measurements of the sizes and settling of oceanic particulates." J. Geophys. Res., 87: 5681-5685

7. Heflinger L.O., Stewart G.L., Booth C.R. (1978) "Holographic motion pictures of microscopic plankton" Applied Optics 17: 951-954

8. Hobson, P.R. (1988) "Precision coordinate measurements using holographic recording." J.Phys.E.: Sci. Inst., 21: 138-145

9. Meng H., et al, (1993) "Intrinsic speckle noise in in-line holography", J.opt.Soc.Am., 10 :2046-2058

10. Xiao Fang, Hobson P.R. (1998) "Effect of spherical aberration on realimage fidelity from replayed in-line holograms of underwater objects" Appl Opt, 37 3206-3214

11. Kilpatrick, J.M. \& Watson, J. (1994) "Precision replay of underwater holograms" Meas Sci Technol 5: 716-725

12. Watson J., Hobson P.R., Krantz E., Lampitt R.S. \& Rogerson A, (1995) "Holographic mensuration of suspended particles in aquatic systems", Proc.SPIE 2577: 191-199 